\title{
Association of Job Strain With Cortisol and Alpha-Amylase Among Shift-Working Health Care Professionals in Laboratory and Field
}

Karhula, Kati

2016-01

Karhula , K, Harma , M , Sallinen, M , Lindholm , H, Hirvonen , A, Elovainio , M , Kivimaki , M , Vahtera , J \& Puttonen , S 2016, ' Association of Job Strain With Cortisol and Alpha-Amylase Among Shift-Working Health Care Professionals in Laboratory and Field ' , Biological Research for Nursing , vol. 18 , no. 1 , pp. 101-112 . https://doi.org/10.1177/1099800415577801

http://hdl.handle.net/10138/223847

https://doi.org/10.1177/1099800415577801

publishedVersion

Downloaded from Helda, University of Helsinki institutional repository.

This is an electronic reprint of the original article.

This reprint may differ from the original in pagination and typographic detail.

Please cite the original version. 


\title{
Association of Job Strain With Cortisol and Alpha-Amylase Among Shift-Working Health Care Professionals in Laboratory and Field
}

\author{
Kati Karhula, PhD ${ }^{1,2}$, Mikko Härmä, MD', Mikael Sallinen, PhD $^{1,3}$, \\ Harri Lindholm, MD ${ }^{4}$, Ari Hirvonen, PhD $^{4}$, Marko Elovainio, PhD ${ }^{5}$, \\ Mika Kivimäki, $P D^{1,2,6}$, Jussi Vahtera, $\operatorname{PhD}^{7,8}$, and \\ Sampsa Puttonen, PhD ${ }^{1,2}$
}

\begin{abstract}
Although the prevalence of work-related stress has increased, knowledge on the contributions of that stress to long-term adverse health effects is still lacking. Stress biomarkers can reveal early signs of negative health effects, but no previous studies have measured both acute stress reactions and long-term exposure to job strain using both salivary cortisol and $\alpha$-amylase (AA). The present study examines the association between job strain and these biomarkers among shift-working female health care professionals in the laboratory and the field. The 95 participants were recruited from hospital wards categorized in either the top (high job strain [HJS] group, $n=42$ ) or the bottom quartile of job strain (low job strain [LJS] group, $n=53$ ), as rated by survey responses. Participants' self-perceived job strain was at least as high or low as the ward's average estimation. Saliva samples were collected during the Trier Social Stress Test (TSST), preselected morning and night shifts, and a day off. There was a larger increase in the cortisol concentration of participants in the HJS than in the LJS group (2.27- vs. I.48-fold, respectively, nonsignificant) during the TSST. Participants in the HJS group also had higher salivary AA levels 30 min after awakening on the morning-shift day than those in the LJS group $(p=.02)$, whereas the salivary cortisol awakening response on the day off was higher in the LJS group ( $p=.05$, education as a covariate). The remaining stress-biomarker results did not differ significantly between groups. These data suggest that HJS in shift-working health care professionals is weakly associated with changes in stress biomarkers.
\end{abstract}

\section{Keywords}

work-related stress, stress biomarkers, nursing, night-shift work

Work-related stress is on the rise and currently affects a fourth of all employees in Europe. Similarly, sickness absence due to work-related stress has increased (Houdmont, Kerr, \& Addley, 2012). High-strain work (high demands combined with low control over work content) may, in the long term, adversely affect health and increase the risk of various diseases. Employees with job strain are at an approximately $20 \%$ higher risk of coronary heart disease than those free of job strain (Kivimäki et al., 2012). Job strain may affect the risk of cardiovascular disease indirectly, through unhealthy behaviors such as smoking and physical inactivity, or directly, by activating neuroendocrine responses to stressors, or both (Chandola et al., 2008). Occupational stress is frequently a factor in the etiology of high ambulatory blood pressure (Rosenthal \& Alter, 2012). Moreover, job strain is associated with decreased heart rate variation (Chandola et al., 2008), which is an independent risk factor for morbidity and mortality (Thayer, Yamamoto, \& Brosschot, 2010).

\footnotetext{
' Development of Work and Organizations, Finnish Institute of Occupational Health, Helsinki, Finland

${ }^{2}$ Institute of Behavioural Sciences, University of Helsinki, Helsinki, Finland

${ }^{3}$ Agora Center, University of Jyväskylä, Jyväskylä, Finland

${ }^{4}$ Health and Work Ability, Finnish Institute of Occupational Health, Helsinki, Finland

${ }^{5}$ National Institute of Health and Welfare, Helsinki, Finland

${ }^{6}$ Department of Epidemiology and Public Health, University College London, London, United Kingdom

${ }^{7}$ Development of Work and Organizations, Finnish Institute of Occupational Health, Turku, Finland

${ }^{8}$ Department of Public Health, University of Turku and Turku University Hospital, Turku, Finland
}

\section{Corresponding Author:}

Kati Karhula, PhD, Development of Work and Organizations, Finnish Institute of Occupational Health, Topeliuksenkatu 4la A, 00250 Helsinki, Finland.

Email: kati.karhula@ttl.fi 
Previous studies of job strain have been conducted mainly among daytime workers (Kompier, Taris, \& van Veldhoven, 2012). Among shift workers, long-term stress may increase the probability of a comanifestation of several individual health risk factors, such as sedentary lifestyle and high blood pressure and cholesterol levels that, in the long term, predispose to chronic disease (Härmä, Kompier, \& Vahtera, 2006). Differences in shift ergonomics are also associated with job strain (Karhula et al., 2013).

There are three primary models of work stress, namely, the effort-reward imbalance model (Siegrist, 1996), the organizational injustice model (Elovainio, Kivimäki, \& Vahtera, 2002), and the job-strain model (Karasek \& Theorell, 1990). The jobstrain model is one of the most influential work-stress models because it defines job demands (JDs) and job control (JC) as the most important determinants of work-related well-being and health (Lindfors et al., 2007). The model suggests that JC modifies the potentially adverse health effects of JDs (Karasek et al., 1998).

Knowledge regarding exactly how stress contributes to well-documented long-term adverse health effects and the evidence on underlying mechanisms are still lacking. Stress biomarkers can reveal early signs of negative health effects, but few studies have explored work stress, specifically, and stress biomarkers.

The main axes of neuroendocrine responses to stress are the autonomic nervous system (ANS) and the hypothalamic-pituitary-adrenal (HPA) axis. The most common psychophysiological stress biomarkers include sympathoadrenal biomarkers such as adrenaline and noradrenaline and the HPA axis biomarker cortisol (Chandola, Heraclides, \& Kumari, 2010). Cortisol can be measured noninvasively and reliably from saliva samples (King \& Hegadoren, 2002; Woods \& Mentes, 2011) and is therefore used extensively in psychoneuroendocrine research (Woods \& Mentes, 2011). In healthy humans, the diurnal cortisol rhythm reaches a substantial peak after awakening and low levels in the evening (Fries, Dettenborn, \& Kirschbaum, 2009). The typical cortisol awakening response (CAR) shows a $50-60 \%$ increase in cortisol concentration (Chandola et al., 2010) in the first 30-45 min after awakening (Nater, Skoluda, \& Strahler, 2013). Studies of cortisol profiles during stress have given somewhat conflicting results (Wong, Ostry, Demers, \& Davies 2012). In a review of CAR and psychosocial factors, the authors found that increased CAR was associated with both job strain and general life stress (Chida \& Steptoe, 2009). Long-term high work stress is also associated with elevated evening cortisol levels (Rydstedt, Cropley, Devereux, \& Michalianou, 2008).

Salivary alpha-amylase (sAA) is a sympathetic nervous system stress marker that has rapid stress responsiveness. Although sAA has not been studied as extensively as cortisol (Rohleder \& Nater, 2009), it seems to be a valid and reliable stress marker (Nater et al., 2006). The normal diurnal sAA profile has a dip after awakening. There is evidence that exposure to long-term stress is associated with flatter diurnal slopes and decreased daily production of sAA (Rohleder \& Nater, 2009).
However, only a few studies have examined work stress and sAA (Groer et al., 2010; Limm et al., 2011; Wingenfeld et al., 2010; Wong et al., 2012), and none of them have included both a valid job-strain measure and real-life work conditions. One of the studies (Wingenfeld et al., 2010) found that sAA profiles of day-shift nurses were not associated with work stress.

The results on the relationship of shift characteristics with stress biomarkers are ambiguous. Some findings suggest that night work in particular is associated with elevated cortisol secretion (Thomas, Hertzman, \& Power, 2009), although Federenko et al. (2004) reported greater CARs for nurses working morning shifts than nurses working evening or night shifts. When examining these studies, it is important to note that stress-biomarker results and self-reports of stress may not be consistent. For example, in one study nurses in intensive care units reported worse fatigue, anxiety, and depressive mood than those in general hospital wards, but researchers found no corresponding differences in cortisol levels (Fujimaru et al., 2012).

Because the results for salivary cortisol and sAA in stress studies have been inconsistent, there is a need for new, sensitive biological stress indicators. One promising method for analyzing biomarker data is to calculate the ratio of sAA to cortisol or vice versa. Ali and Pruessner (2012) suggest that, as the ratio of sAA over cortisol (AOCg) reflects both sympathetic nervous system and HPA axis function, it is a better indicator of stress system dysregulation than either of these biomarkers alone. Further, the authors report a strong correlation between $\mathrm{AOCg}$ and self-reported indexes of stress, which has not always been the case in studies measuring HPA axis function and behavior. In a recent review article, Andrews, Ali, and Pruessner (2013) also recommend the use of these biomarker ratios.

Researchers have generally assumed that stress studies conducted in the laboratory reflect the way in which individuals react to stressors in their everyday lives (Kidd, Carvalho, \& Steptoe, 2014). According to this assumption, an individual who is highly reactive in the laboratory would be prone to experiencing repeated episodes of heightened arousal in her or his real life that will subsequently have an impact on health risks (Steptoe, 2007). However, few studies have actually assessed the associations between an individual's cortisol responses in the laboratory and those occurring in daily life. To our knowledge, no previous studies have included laboratory-field paradigms with sAA measures. More specifically, no previous studies have measured the effects of both acute stress reactions in the laboratory and longer term exposure to a stressful work environment on both salivary cortisol and sAA.

In the present study, we aimed to examine the extent to which exposure to job strain is associated with stress biomarkers among shift workers in both laboratory and field conditions. Study settings were a standardized laboratory condition when participants were not under acute workload stress or circadian disruption and work shifts in natural working conditions. Our hypotheses were that exposure to high job strain (HJS) would be associated with augmented acute salivary 
cortisol and sAA responses and a smaller sAA to cortisol ratio compared to low job strain (LJS) in the laboratory; while in the field, we expected HJS to be associated with a blunted CAR, elevated evening cortisol levels, and decreased total sAA secretion in connection with work shifts. We also explored whether the individuals with augmented cortisol reactions in the laboratory showed elevated CARs and evening cortisol levels in daily life. In addition to the unique study design combining laboratory and field studies in the same individuals, the main strength of this study was our use of the average work unit estimation of job strain to reduce subjectivity bias, such as negative affectivity (Rugulies, 2012), for testing the association between job strain and stress biomarkers.

\section{Method}

\section{Study Sample}

We recruited participants from among the 2008 Finnish Public Sector Study (FPSS) survey respondents $(N=52,891$, response rate $71.5 \%$ ). From this sample, we identified native Finnishspeaking female health care professionals working night shifts ( $n=5,615$; Figure 1). The sample included five hospitals or health care districts and four cities in southern Finland. The participants were from 59 wards, which had an average of 37 employees.

We sent an invitation letter to the workplace of participants fulfilling the following inclusion criteria according to their FPSS survey responses $(n=422)$ : age $30-58$ years, body mass index (BMI) under $35 \mathrm{~kg} / \mathrm{m}^{2}$, and at least 3 years of work experience in the same ward. The age limits were set to ensure adequate exposure to work life and to minimize the proportion of participants who would be retiring during the study. Those with coronary heart disease or insulin-treated diabetes mellitus, who had a disease or were taking a medication affecting cognitive functions, or who were pregnant or breast-feeding were excluded. The primary reasons potential participants were not included $(n=65)$ were changing ward or workplace $(n=$ $22)$ or quitting night-shift work $(n=21)$ after the 2008 survey.

Job strain was measured using Karasek's Job Content Questionnaire (JCQ) in the FPSS survey (Karasek et al., 1998). The JCQ has been used in a wide range of work-stress studies among various nations (Karasek et al., 1998; Kivimäki et al., 2012). As mentioned earlier, the job-strain model defines JD and $\mathrm{JC}$ as the most important determinants of work-related well-being and health (Lindfors et al., 2007). Accordingly, we used three of the five questions on the JCQ related to JD (e.g., pace and pressure of work tasks) and nine related to JC (e.g., individual autonomy over working time and methods). Use of at least 3 items from the JD scale has a strong correlation $(r>.90)$ with use of the full scale (Fransson et al., 2012). Respondents rated each item on a 5-point Likert-type scale, ranging from 1 (strongly disagree) to 5 (strongly agree), and we calculated mean JC and JD from these responses.

As described previously (Karhula et al., 2013), we formed the HJS and LJS groups by grouping the wards with at least five

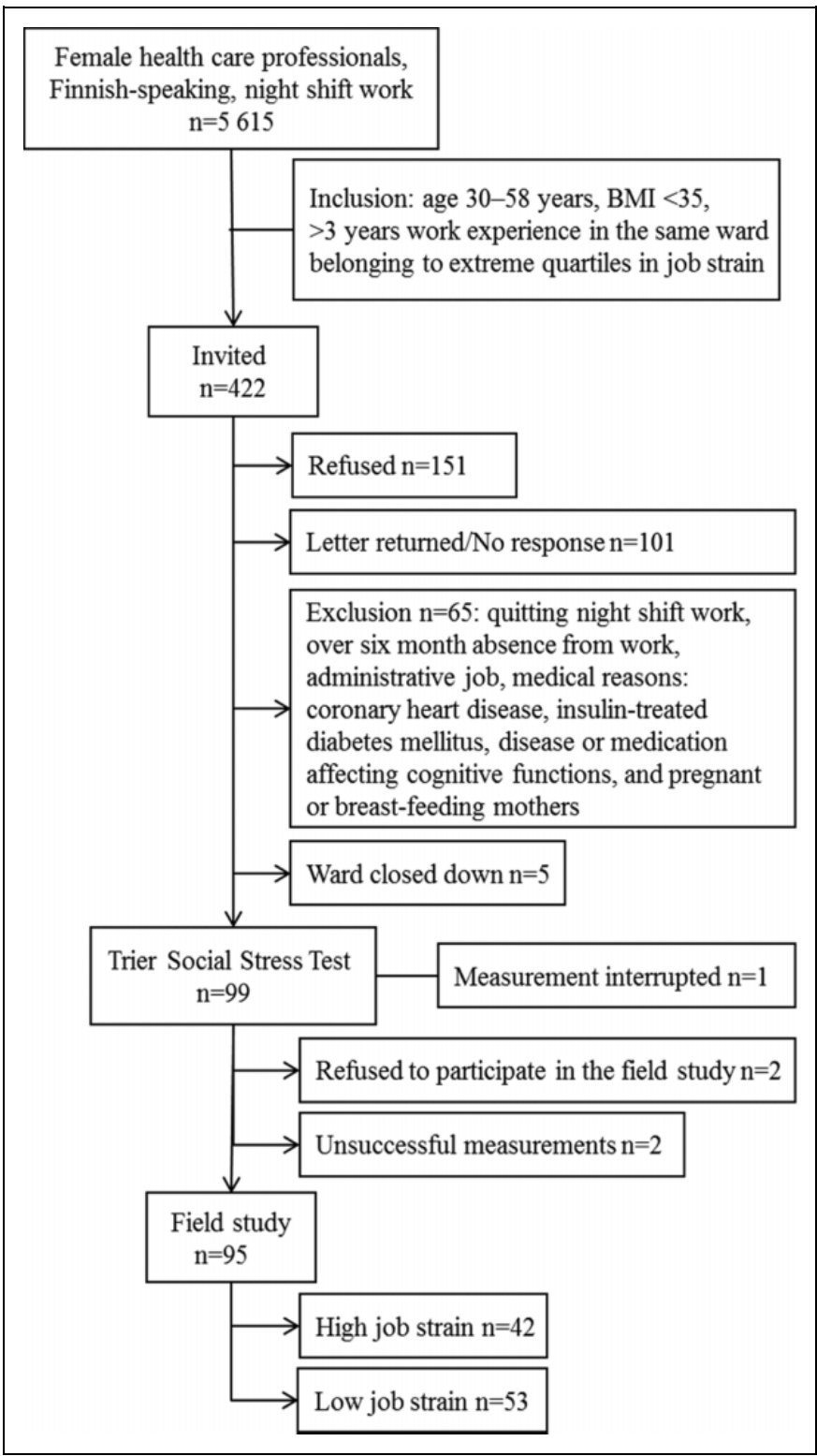

Figure I. Flowchart of the study. BMI = body mass index.

respondents on the JD and JC scales and using a median split to identify HJS (high demands and low control) and LJS (low demands and high control) wards. Using these cutoff points, we identified employees who belonged to the HJS or LJS group also on the basis of their individual mean JD and JC scores. We increased the contrast between the job-strain groups by excluding the employees belonging to the quartile with the least strain in the HJS group $(n=86)$ and the most strain in the LJS group $(n=48)$ from the group of individuals who fulfilled all other inclusion criteria, which gave us our final number of 422 invited to participate, as described earlier. The mean values for $\mathrm{JD} / \mathrm{JC}$ were 4.56/3.20 in the HJS group and 2.64/4.11 in the LJS group (mean difference $1.92 /-0.90, p<.01$ ). The reliability coefficients for JCQ among those invited to participate in the study $(N=422)$ were good (Cronbach's $\alpha=.87$ for JD and .80 for JC). 
We tested the representativeness of the 95 participants by comparing them to the 422 health care workers invited to participate. There were no statistical differences $(p>.11)$ between the 2008 survey responses of those who participated and those who were invited to participate with regard to age, level of education, work experience, number of children, BMI, habitual sleep length, sleep need, or sleep disturbances. There were more participants from the LJS group than the HJS group $(p<.01)$, and fewer of the participants were from the two major health care districts $(p<.01)$ than from the seven smaller health care districts or cities. The participants more often worked in medical-surgical wards than other wards (e.g., intensive care, emergency, or maternity units) compared to the entire population of those invited to participate ( $45 \%$ vs. $33 \%$; $p<.04$ ).

The Coordinating Ethical Committee of the Hospital District of Helsinki and Uusimaa approved the study. We obtained signed informed consent from each participant. The participants were compensated for traveling expenses and given $€ 50$ (approximately US\$64) as compensation for participation.

\section{Procedures}

Laboratory study. The participants arrived at the Finnish Institute of Occupational Health (FIOH, Helsinki, Finland) at 9 a.m., met the research nurse, signed informed consent forms, and completed an Internet-based set of questionnaires for the collection of background information, as described subsequently, and a paper version of Beck's Depression Inventory (BDI-II; Beck, Steer, \& Brown, 1996). We collected a 12-hr fasting blood sample before breakfast. The laboratory day included a baseline saliva sample, administration of cognitive tests (reported elsewhere; see Vuori et al., 2014), and the Trier Social Stress Test (TSST; Kirschbaum, Pirke, \& Hellhammer, 1993) along with corresponding saliva samples and instruction from the research nurse for taking the field measurements.

Field study. During the 3-week field study, we measured participants' sleep patterns and sleep-wake rhythm using a sleep diary and actigraphy (Actiwatch AW7; Cambridge Neurotechnology Ltd, Cambs, United Kingdom). Participants wore the actigraph continuously on the nondominant wrist, except at moments of hygiene concern at the workplace. We analyzed the actigraph data using Actiwatch Activity and Sleep Analysis 7 software. Participants also collected three saliva samples per day on 3 preselected days, as described subsequently.

\section{Measures}

Questionnaires. As part of the laboratory study, we administered an Internet-based questionnaire (Digium QuestBack Company, Espoo, Finland) featuring questions on background information, working conditions, and the duration and number of work shifts and hours. The background variables were BMI (calculated from height and weight measured at the FIOH research laboratory), number of children, level of education, physical activity (times per week the respondent was active for at least
$30 \mathrm{~min} /$ day, during the past 3 months), sleep length, smoking, and alcohol consumption. As part of the Internet-based data collection, we also administered a chronotype questionnaire (Morningness-Eveningness Questionnaire; Smith, Reilly, \& Midkiff, 1989) and asked about stressful life events that had occurred during the preceding 12 months (Dohrenwend, Krasnoff, Askenasy, \& Dorhenwend, 1978). Participants also completed a paper version of the Beck Depression Inventory (BDI-II; Beck et al., 1996). We interviewed participants about the use of prescription medication. Finally, fasting blood glucose, total cholesterol, and low-density lipoprotein (LDL) cholesterol were analyzed from the blood samples that were collected at the FIOH research laboratory.

TSST. We conducted the TSST (Kirschbaum et al., 1993) at FIOH as part of the laboratory study at approximately 1 p.m. Participants were instructed to play the role of a job applicant in an interview for a research nurse's job and given 5 min to prepare a 5-min speech. They delivered the speech to one unknown assessor. After the speech, the research nurse asked the participant to orally serially subtract the number 13 from 2083 as fast and as accurately as possible for $5 \mathrm{~min}$. The research nurse debriefed participants about the purpose of the test.

Salivary cortisol and AA. During the laboratory study, the first saliva sample (baseline) was collected at approximately 10:30 a.m. after collection of fasting blood sample, filling in questionnaires and a rest period following participants' arrival at FIOH at 9 a.m. We collected the TSST1 saliva sample at the beginning of the TSST, the TSST2 saliva sample immediately after the test was finished, and the TSST3 saliva sample $15 \mathrm{~min}$ after the TSST. The collection of saliva samples and their analysis succeeded in $99.1 \%$ of cortisol and $98.9 \%$ of AA samples. One participant was excluded from all analyses due to medication for rheumatic disease, and two participants were excluded from the analysis of the cortisol results due to asthma medication including cortisone with a long-term effect and inexplicable high cortisol values.

During the field study, saliva collection took place 3 times a day on 3 preselected, nonconsecutive days (i.e., 24-hr period), including a day on which the participant worked a morning shift (mostly 07:00-15:00), a day on which she worked a night shift (mostly 21:00-07:00), and a day off. To optimize the similarity in circadian rhythm and recovery between participants, the specific criteria for the preselected measurement days were (1) at least the third consecutive morning shift, (2) the first night shift after a morning or evening shift, and (3) the second consecutive day off.

For the measurement of the CAR, participants collected saliva samples immediately after waking up (AW) and $30 \mathrm{~min}$ after waking up (30AW). They collected the third sample before brushing their teeth and going to sleep, preferably around 10 p.m. on the morning-shift day and day off and around 8 a.m. on the night-shift day. Participants refrained from eating or drinking between awakening and the AW and 
AW30 sample collection and for at least $15 \mathrm{~min}$ before the evening sample collection. They were instructed to rinse their mouth several times with water before collection of the evening sample. The Salivette tubes were marked with a different sticker color for each measurement day, with numbers 1-3 corresponding with each sample and with a space for writing down the exact time of sample collection. Participants stored the saliva samples in their home refrigerators and mailed them to FIOH by regular post, as saliva samples tolerate the prevailing temperatures during shipment (Andrews, Ali, \& Pruessner, 2013; Clements \& Parker, 1998; Rohleder \& Nater, 2009). One participant was excluded from both cortisol and sAA analyses due to a failure in sample storage.

All the saliva samples were collected using Salivette tubes and cotton rolls, which the participants placed in their mouths for at least $1 \mathrm{~min}$ and then replaced into the Salivette tube. The saliva samples were analyzed in FIOH's laboratories using a chemiluminescence immunoassay LIA kit (LIA; IBL, Hamburg, Germany) with a measurement range of 0.43-110 $\mathrm{nmol} / \mathrm{L}$. A greater than $50 \%$ increase in cortisol concentration over baseline was considered a high cortisol response for the laboratory study samples. In the analysis of the field study samples, the limit for high CAR was set at a greater than $60 \%$ increase in cortisol concentration from the AW sample to the AW30 sample (Pruessner et al., 1997). Salivary AA was analyzed using the sAA kinetic enzyme assay kit (Item No. 1-1902; Salimetrics, Carlsbad, CA, USA). Samples between 2.0 and $900 \mathrm{U} / \mathrm{ml}$ were accepted (Wong et al., 2012).

\section{Statistical Analysis}

Statistical analyses were conducted using PASW 18.0 (formerly SPSS; Predictive Analytics Software, Chicago, Illinois). The independent samples $t$-test was used to compare the stress groups for continuous background variables, for example, age and shiftwork experience. The Pearson's chi-square test was used to determine group differences for categorical variables, for example, chronotype and family caregiving responsibility. Fisher's exact test was used for dichotomous variables in $2 \times 2$ tables.

Logarithmic transformations were carried out for the skewed biomarker distributions. One-way analysis of variance (ANOVA) was used to explore the differences between the job-strain groups in stress biomarker levels. The general linear model ANOVA for repeated measures was used to test stress biomarker profiles. The area under the curve with respect to the ground $(\mathrm{AUCg} \mathrm{nmol} / \mathrm{l} \times \mathrm{min}$; Pruessner, Kirschbaum, Meinlschmid, \& Hellhammer, 2003) was calculated assuming linearity between the consecutive points in the analysis of the total secretion of the stress biomarkers. The sAA to cortisol ratios were calculated from the AUCg results (Ali \& Pruessner, 2012).

As there was were significant differences in the levels of education and physical activity between job-strain groups, these variables were used as covariates in the analysis. A $p$ value of $<.05$ indicated a statistically significant result throughout the study.

\section{Results \\ Participants}

Of the 95 participants, 42 (44\%) belonged to the HJS group and $53(56 \%)$ to the LJS group. The participants most often worked in medical-surgical wards $(45 \%, n=43)$ and intensive care or emergency units $(15 \%, n=14)$. Their mean age was 47 years (min-max 31-59 years), with no significant difference in age between the job-strain groups. Number of working hours, sleep duration, shift-work experience, family caregiving responsibility, stressful life events, chronotype, and sickness absence during the field measurements also showed no significant differences by stress group (Table 1). Similarly, there were no statistical differences between the groups in the number of work shifts, night shifts, or over 12-hr work shifts or in lifestyle factors (BMI, fasting blood glucose, total and LDL cholesterol, smoking, alcohol consumption, or medication use).

The proportion of nurses versus nursing assistants was higher in the LJS group (81\%) than in the HJS group $(60 \%$; $p=.02$ ). The LJS group reported higher levels of physical activity than the HJS group $(p<.01)$. The average number of working hours per 3 weeks was $111 \mathrm{hr}, 45 \mathrm{~min}$ for the whole sample.

\section{Salivary Sample Collection}

The mean saliva sample collection times for the field study were 05:27 (AW), 05:57 (AW30), and 22:36 for the morning-shift day; 08:08 (AW), 08:41 (AW30), and 08:06 the next morning for the night-shift day; and 07:56 (AW), 08:21 (AW30), and 21:34 for the day off. The AW and AW30 samples were collected on average 6 and 35 min after sleep end (actigraphically determined) on the morning-shift day, 8 and $38 \mathrm{~min}$ after sleep end on the night-shift day, and 6 and $35 \mathrm{~min}$ after sleep end on the day off.

\section{Acute Salivary Cortisol and AA Stress Reactivity}

Salivary cortisol and AA levels were similar between groups at baseline in the laboratory study (Table 2). The TSST caused an average 2.27 -fold increase in salivary cortisol concentration in the HJS group and an average 1.48-fold increase in the LJS group. There were no significant differences in salivary cortisol or AA levels between job-strain groups at any time point in the laboratory study.

The profiles of salivary cortisol and AA showed no jobstrain group differences during or after the TSST. The total secretion of salivary cortisol and AA calculated as the AUCg was similar on the TSST and the laboratory measurement day (baseline + TSST) in both job-strain groups $(p>.22$, data not shown).

\section{CAR and Evening Cortisol Values in the Field}

The magnitude of CAR was $51 \%$ in the HJS group and $50 \%$ in the LJS group on the morning-shift day and $31 \%$ and $30 \%$, 
Table I. Background Variables by Job-Strain Group.

\begin{tabular}{|c|c|c|c|c|c|}
\hline Variable & $\begin{array}{l}\text { Low Job Strain } \\
(n=53) \\
\text { Mean }(S D)\end{array}$ & $\begin{array}{l}\text { High Job Strain } \\
\quad(n=42) \\
\text { Mean (SD) }\end{array}$ & $d f$ & $t$-Value & $p$ Value $^{\mathrm{a}}$ \\
\hline BMI, $\mathrm{kg} / \mathrm{m}^{2}$ & $25.6(4.0)$ & $26.7(4.1)$ & 93 & 1.29 & .20 \\
\hline Shift-work experience, years & $16.9(7.6)$ & I8.7 (7.9) & 90 & 1.11 & .27 \\
\hline Sleep duration, hr, actigraphy & $6: 50(0: 40)$ & $6: 46(0: 43)$ & 93 & 0.57 & .57 \\
\hline \multirow[t]{2}{*}{ Low-density lipoproteins, mmol/L } & $2.9(0.6)$ & $3.2(0.8)$ & 93 & 1.83 & .07 \\
\hline & $n(\%)$ & $n(\%)$ & \multicolumn{2}{|r|}{$\chi^{2}$ value } & $p$ Value \\
\hline Education & & & \multicolumn{2}{|r|}{5.38} & $.02^{\mathrm{b}}$ \\
\hline Nurse $^{c}$ & $43(81)$ & $25(60)$ & & & \\
\hline Family caregiving responsibilityg & & & \multicolumn{2}{|r|}{0.44} & $.5 \mathrm{I}^{\mathrm{b}}$ \\
\hline Yes & 31 (69) & $21(62)$ & & & \\
\hline No & $14(3 \mid)$ & $13(38)$ & & & \\
\hline Stressful life events during past year & & & \multicolumn{2}{|r|}{0.34} & $.34^{b}$ \\
\hline Yes & $39(75)$ & $27(66)$ & & & \\
\hline No & $13(25)$ & $14(34)$ & & & \\
\hline Chronotype $^{\mathrm{h}}$ & & & \multicolumn{2}{|r|}{0.58} & $.58^{\mathrm{b}}$ \\
\hline Morning type & $27(5 I)$ & $19(45)$ & & & \\
\hline Evening type & $26(49)$ & $23(55)$ & & & \\
\hline Prescription medication use $\mathrm{e}^{\mathrm{i}}$ & & & \multicolumn{2}{|r|}{0.41} & $.52^{\mathrm{b}}$ \\
\hline Yes & $30(57)$ & $21(50)$ & & & \\
\hline No & $23(43)$ & $21(50)$ & & & \\
\hline
\end{tabular}

Note. $\mathrm{BMI}=$ body mass index.

${ }^{a}$ Independent samples $t$ test. ${ }^{\mathrm{b}}$ Pearson's chi-square. ${ }^{\mathrm{C}} \mathrm{Nurse}$, midwife, public-health nurse, $\mathrm{X}$-ray nurse, deaconess nurse. ${ }^{\mathrm{d}} \mathrm{Nursing}$ assistant, practical nurse, nursery

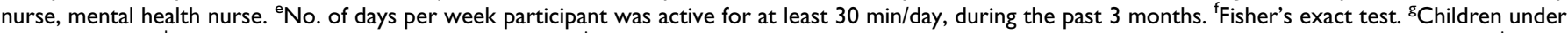
18 years of age. 'Morningness-Eveningness Questionnaire. 'Self-reported medications including oral contraceptives and hormone-replacement therapy. 'Beck's Depression Inventory-II score $\geq 19 / 63$.

respectively, on the night-shift day $(p>.76)$, but on the day off, the values showed a greater difference at $32 \%$ and $40 \%$, respectively ( $p=.17$ and $p=.05$ with education as a covariate; Figure 2). The evening declines were also similar between the stress groups. In the unadjusted analyses, there were no stress-group differences in salivary cortisol levels at $\mathrm{AW}$, at $30 \mathrm{AW}$, or before going to sleep on the morning shift, night shift, or day off day (Table 3).

\section{$s A A$ Levels and Profiles in the Field}

sAA levels were higher in the HJS group at $30 \mathrm{AW}$ on the morning-shift day (Figure 2). There were no other significant between-group differences in average sAA levels during the morning-shift, night-shift, or day off measurement days (Table
3). In both job-strain groups, there was a similar dip in sAA levels after waking up $(p \geq .14)$. A similar elevation in the evening levels was also detected in both groups.

\section{Total Secretion of Salivary Cortisol and AA}

The total secretion of salivary cortisol and sAA, measured as AUCg, was similar in the two job-strain groups $(p>.25$; data not shown) for the measurement days.

\section{Stress-Biomarker Ratios}

The unadjusted sAA over cortisol and cortisol over sAA ratios from the AUCg results were similar for the two job-strain groups ( $p>.40$ and .55 , respectively; Table 4$)$. 
Table 2. Salivary Cortisol and Alpha-Amylase Levels in the Laboratory Study With TSST by Job-Strain Group.

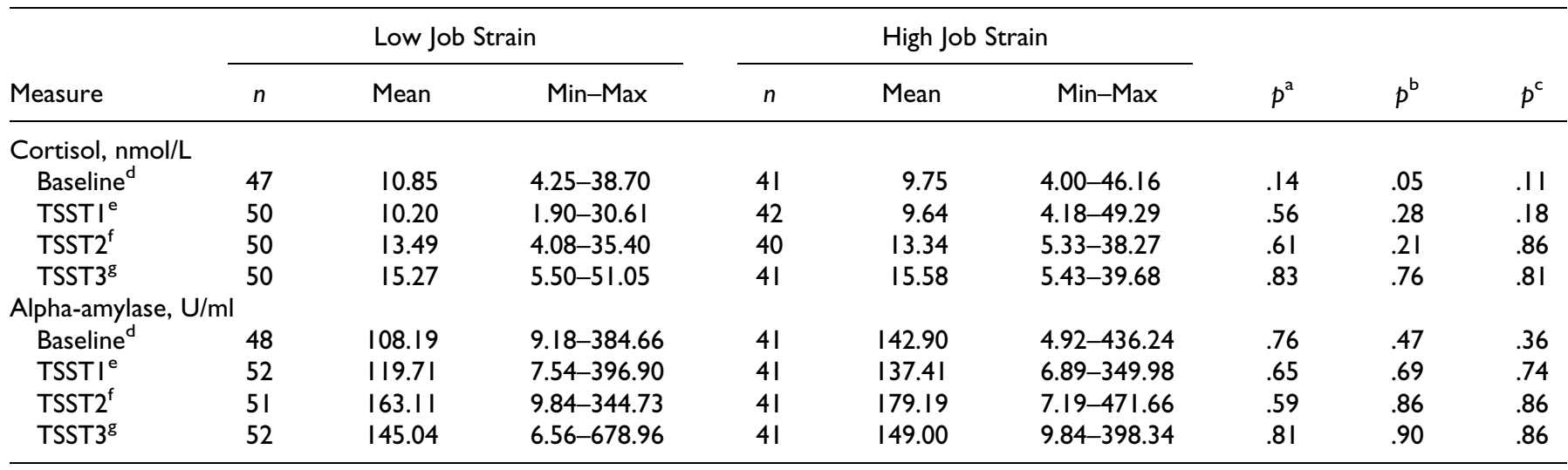

Note. Baseline $=$ after arrival at the laboratory; TSST $=$ Trier Social Stress Test; TSSTI $=$ at the beginning of the TSST; TSST2 = at the end of the TSST; TSST3 = 15 min after completing the TSST.

${ }^{a}$ Analysis of variance for log-transformed distribution. ${ }^{b}$ Education as a covariate. ${ }^{c}$ Physical activity as a covariate. ${ }^{d}$ After arriving to the laboratory. ${ }^{e}$ At the beginning of the TSST. ${ }^{f} A t$ the end of the TSST. ${ }^{8} \mid 5$ minutes after completing the TSST.

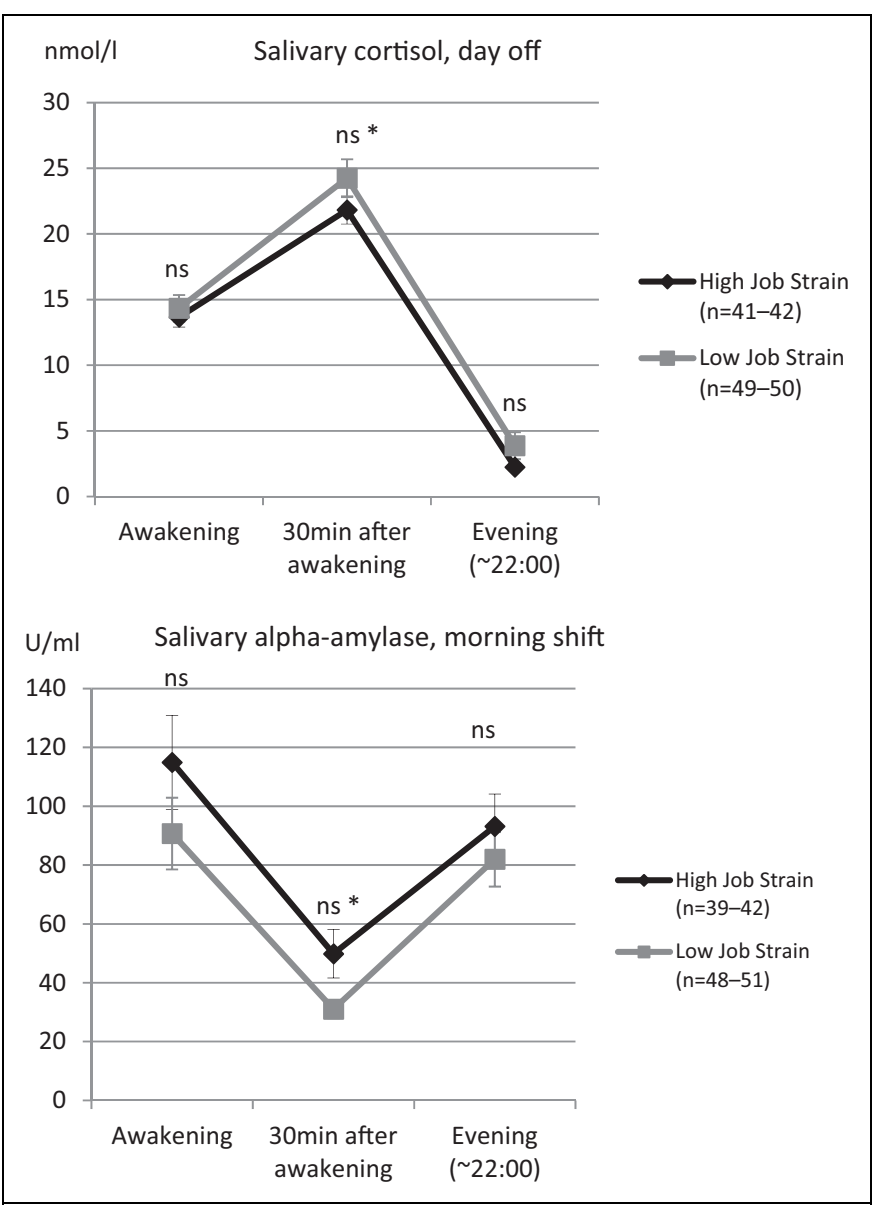

Figure 2. Salivary cortisol and alpha-amylase profiles on measurement days with significant job-strain group differences. Vertical lines represent standard error of mean (SEM). ns = nonsignificant statistical difference between job-strain groups. *Significant with education as a covariate.

\section{Discussion}

This study adds to the limited literature on the associations between work stress and stress biomarkers. We found that sAA levels were higher in the HJS group than in the LJS group $30 \mathrm{AW}$ on the morning-shift day. A larger proportion of the LJS group had a high cortisol response to TSST and elevated CAR on the morning-shift day. The CAR on the day off was higher in the LJS group than in the HJS group. The rest of the salivary biomarker levels, profiles, total secretion, and biomarker ratios showed largely similar results across the two job-strain groups.

To our knowledge, this is the first study to examine the associations between job-strain and salivary cortisol and AA responses among shift workers in both laboratory and field. Moreover, we are not aware of any previous studies using the TSST among health care staff. There was a larger increase in the salivary cortisol concentration in the TSST in the HJS group than in the LJS group, but the difference was not statistically significant. Similarly, researchers in one previous study found no difference in the cortisol responses to the TSST between employees with burnout and healthy employees (De Vente, Olff, Van Amsterdam, Kamphuis, \& Emmelkamp, 2003). The TSST induces profound endocrine responses in the majority of people, and these responses are, on average, higher among males than females (Kirschbaum et al., 1993). It may thus be more difficult to observe group differences in the response to the test among females.

Job strain was not associated with the salivary cortisol levels or profiles in the field. In concordance with our results, Fujiwara et al. (2004) found that HJS and LJS groups among health care staff had similar salivary cortisol concentrations on both a night-shift day and a day off. However, in a study by Steptoe, Cropley, Griffith, and Kirschbaum (2000), HJS among teachers was associated with significantly elevated salivary cortisol on the morning of a work day compared to the teachers with LJS. In a previous study, we found job-strain group differences in perceived workload and intershift recovery (Karhula et al., 2013), but these differences were not manifested in the stress biomarkers. The findings on biomarkers and self-reported stress are inconsistent (Fujimaru et al., 2012; Fujiwara et al., 2004), while recovery and cortisol results are more concordant (Gustafsson, Lindfors, Aronsson, \& Lundberg, 2008). 
Table 3. Salivary Cortisol (nmol/L) and Alpha-Amylase Levels (U/ml) During Field Measurements.

\begin{tabular}{|c|c|c|c|c|c|c|c|c|c|}
\hline Measure & \multicolumn{3}{|c|}{ Low Job Strain } & \multicolumn{3}{|c|}{ High Job Strain } & $p^{\mathrm{a}}$ & $p^{b}$ & $p^{c}$ \\
\hline \multicolumn{10}{|l|}{ Morning shift } \\
\hline AW & 49 & 10.41 & $(2.57-33.66)$ & 41 & 11.14 & $(5.45-20.10)$ & .18 & .19 & .50 \\
\hline 30AW & 49 & 22.86 & $(6.67-48.48)$ & 42 & 24.98 & $(6.17-58.48)$ & .23 & .40 & .61 \\
\hline AW & 49 & 12.81 & $(1.20-26.25)$ & 41 & 12.83 & $(5.24-27.57)$ & .64 & .72 & .10 \\
\hline 30AW & 50 & 21.22 & $(1.04-53.79)$ & 41 & 20.31 & $(6.56-42.74)$ & .64 & .21 & .47 \\
\hline Before sleep (08:00) & 49 & 11.84 & $(0.73-31.75)$ & 42 & $1 \mathrm{I} .57$ & $(4.64-18.44)$ & .84 & .03 & .31 \\
\hline \multicolumn{10}{|l|}{ Day off } \\
\hline AW & 50 & 14.33 & $(3.54-33.15)$ & 42 & 13.66 & $(6.46-29.04)$ & .92 & .78 & .44 \\
\hline 30AW & 49 & 24.24 & $(8.58-53.95)$ & 42 & 21.82 & $(10.84-40.56)$ & .23 & .12 & .20 \\
\hline 30AW & 48 & 31.02 & $(6.23-119.06)$ & 39 & 49.83 & $(3.61-269.62)$ & .16 & .06 & .38 \\
\hline Before sleep (22:00) & 51 & 81.97 & $(2.30-266.66)$ & 42 & 93.16 & $(2.95-272.57)$ & .40 & .67 & .35 \\
\hline \multicolumn{10}{|l|}{ Night shift } \\
\hline AW & 52 & 79.77 & $(2.95-357.52)$ & 39 & 92.88 & $(3.6 I-386.06)$ & .94 & .88 & .19 \\
\hline 30AW & 50 & 43.08 & $(5.58-161.70)$ & 40 & 52.60 & $(6.89-168.59)$ & .31 & .37 & .27 \\
\hline Before sleep (08:00) & 51 & 88.63 & $(2.95-596.96)$ & 42 & 80.98 & $(3.6 I-366.05)$ & .59 & .79 & .74 \\
\hline \multicolumn{10}{|l|}{ Day off } \\
\hline AW & 51 & 78.20 & $(4.43-248.05)$ & 41 & 112.46 & $(3.94-487.4 I)$ & .44 & .75 & .20 \\
\hline 30AW & 49 & 38.51 & $(2.62-105.62)$ & 41 & 46.34 & $(2.95-193.85)$ & .53 & .42 & .76 \\
\hline Before sleep (22:00) & 52 & 117.28 & $(3.61-347.68)$ & 42 & 110.42 & $(5.90-457.88)$ & .62 & .70 & .29 \\
\hline
\end{tabular}

Note. $\mathrm{AW}=$ awakening; $30 \mathrm{AW}=30 \mathrm{~min}$ after awakening; $\mathrm{ANOVA}=$ analysis of variance.

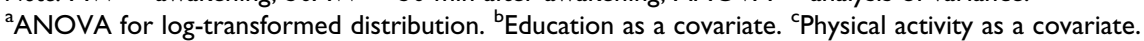

Table 4. Salivary Alpha-Amylase (sAA) Over Cortisol and Cortisol Over Alpha-Amylase Ratios.

\begin{tabular}{|c|c|c|c|c|c|c|c|c|c|}
\hline \multirow[b]{2}{*}{ Ratio } & \multicolumn{3}{|c|}{ Low Job Strain } & \multicolumn{3}{|c|}{ High Job Strain } & \multirow[b]{2}{*}{$p^{a}$} & \multirow[b]{2}{*}{$p^{\mathrm{b}}$} & \multirow[b]{2}{*}{$p^{c}$} \\
\hline & $n$ & Mean & (Min-Max) & $n$ & Mean & (Min-Max) & & & \\
\hline TSST & 53 & 1.27 & $(0.00-1.70)$ & 41 & 1.33 & $(0.98-1.75)$ & .75 & .33 & .80 \\
\hline Morning shift & 45 & 1.13 & $(0.90-1.28)$ & 36 & 1.14 & $(0.8 I-1.3 I)$ & .60 & .52 & .54 \\
\hline Night shift & 49 & 1.12 & $(0.79-1.51)$ & 39 & 1.12 & $(0.82-1.26)$ & .89 & .70 & .85 \\
\hline TSST & 49 & 0.74 & $(0.59-1.01)$ & 41 & 0.75 & $(0.57-1.02)$ & .55 & .25 & .92 \\
\hline Morning shift & 45 & 0.89 & $(0.78-1.11)$ & 36 & 0.88 & $(0.77-1.24)$ & .73 & .23 & .22 \\
\hline Night shift & 49 & 0.91 & $(0.66-1.26)$ & 39 & 0.90 & $(0.80-1.22)$ & .75 & .21 & .42 \\
\hline Day off & 49 & -0.62 & $(-1.44-0.69)$ & 39 & -0.65 & $(-1.40-0.59)$ & .71 & .03 & .15 \\
\hline
\end{tabular}

Note. TSST $=$ Trier Social Stress Test. Ratios were calculated using the area under the curve with respect to the ground.

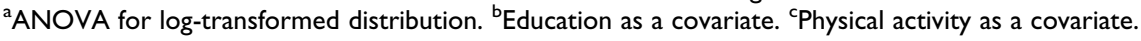

The salivary CARs were lower in both job-strain groups on the morning of a night-shift day and a day off than on a morning-shift day. Earlier research has also shown greater CARs in morning shifts than in late-day shifts or night shifts (Federenko et al., 2004). Anticipation of the next day is of major relevance to the magnitude of the CAR (Fries et al., 2009). In nursing, morning shifts are regarded as the busiest, and both job-strain groups might have anticipated a morning shift that involved time pressure. On the other hand, in our data, the CARs were below the reference values on the night-shift day (Clow, Thorn, Evans, \& Hucklebridge, 2004) and on the day off (Fries et al., 2009), which may be an indication of blunted CAR in both job-strain groups.

The finding that both a high CAR and a high TSST cortisol response occurred more often in the LJS than in the HJS group may indicate that the strong responses to the TSST do not 
reflect job strain in the same way as a high CAR does in the morning before a work shift. Field measurements seem to better reflect the context-specific acute changes in stress reactivity due to job strain. In order to detect generalized group-level changes in stress responses, larger differences in exposure to job strain or burnout symptoms are probably needed.

sAA levels were higher in the HJS group at $30 \mathrm{AW}$ on the morning-shift day than in the LJS group. In the present study, HJS was not associated with flatter diurnal slopes or decreased daily production of sAA, as it was in a previous study (Wong et al., 2012). Contrary to our hypothesis, the ratio of sAA to cortisol was not associated with the level of job strain. In one previous study, Ali and Pruessner (2012) found an association between the ratio of sAA to cortisol and chronic stress. There are, however, differences between that study sample and the present sample in age, gender, stress exposure, and circadian disruption. More studies are needed to clarify the association of AOCg with stress.

Our findings of only a few differences in stress biomarkers between the job-strain groups may partially reflect the general similarity between the HJS and the LJS groups engaged in the same occupation, which, in this case, involves strenuous night-shift work. Group differences in job strain within a single occupation are typically smaller than those in epidemiological studies across several occupations (Hintsanen et al., 2007; Karasek \& Theorell, 1990). The association of job strain with stress biomarkers became visible in connection with morning shifts in the present study. Our interpretation of this finding is that the effects of job strain on stress biomarkers first become visible in real working situations in which acute stressors, such as time pressure, occur.

This experimental study, using both laboratory and field conditions, was nested within a larger ongoing longitudinal cohort study. One strength of the present study was that the measure of job strain was based on a mean score from the same ward. This resulted in a reduced influence of subjectivity on the measure (Rugulies, 2012) and therefore in a less biased estimate of environmental job strain.

Another strength of our study is that the measurement protocol was similar for all participants with respect to circadian rhythm and recovery time, which has often not been the case in studies that have measured cortisol levels among shift workers. The nursing professionals participating in the present study followed the sampling instructions and collection-time guidelines well, which reduced the likelihood of preanalytic errors. In addition, the working-time data were based on realized rosters instead of commonly used self-report data. To our knowledge, no prior studies have either conducted research on stress reactivity among nursing personnel or combined experimental research and field studies in shift workers in stress biomarker research.

Another important strength of our study is the homogenous study sample, as the participants were all relatively healthy shift-working females who reported similar working hours, family caregiving responsibilities, stressful life events, and occurrence of sickness absence during the field study.
Participants' illnesses and prescription medication use were also well controlled compared to more epidemiological study designs. While saliva samples generally can be collected easily by participants themselves (Kudielka, Gierens, Hellhammer, Wust, \& Schlotz,. 2012), the health care workers in the present study followed the sample collection protocol particularly carefully. All these factors would have likely reduced the bias and confounding of between-group results. There were statistically significant differences between the groups in physical activity and education which, when controlled for, had little effect on results.

The present study also has some limitations. The absolute differences in job strain between the groups of nursing employees in the present study were not as substantial as those seen in earlier studies among different occupational groups, making it relatively difficult to establish clear-cut differences between the groups in stress biomarkers. Although we used exposure data from a prospective data set, the study design for analyzing the effect of job strain on stress biomarkers was cross-sectional, preventing us from drawing conclusions regarding causality. The JCQ specifically assesses job strain and may not capture spillover stress. However, the groups did not differ with respect to family caregiving responsibilities or stressful life events, which should have minimized the differences in spillover stress.

One third of the health care employees that we contacted declined to participate in the study, and only approximately one fourth of the employees who met the inclusion criteria participated. This low participation rate was partly due to lack of personal contact with potential study participants, which meant that we could not verify whether the employees had actually been reached or not. Moreover, the considerable time commitment that the study required most likely also reduced the participation rate. On the other hand, the participants were representative of the pool of invited employees, only a few (4\%) participants refused or failed to follow through with the field measurements, and the biomarker data collection succeeded in $99 \%$ of the samples. It is possible, however, that the individuals most affected by job strain were unwilling to participate, which may have diluted the differences we observed between the job-strain groups.

We used one "staff manager" in the TSST, whereas the standard protocol uses three managers (Kirschbaum et al., 1993). The cortisol responses to the TSST might have been stronger if the test had included more than one evaluator. The fact that we did not collect data on participants' menopausal status is also a potential limitation, as acute sympathoadrenal responsiveness increases after menopause (Kajantie \& Phillips, 2006). Similar to previous studies (Kidd et al., 2014; Wingenfeld et al., 2010; Wong et al., 2012), we had a single measurement day for each shift. It would be preferable to collect saliva samples on several days for each shift type.

\section{Conclusion}

We did not observe strong associations between job strain and stress biomarkers in this sample of health care professionals. Although there was an indication of a higher stress response 
to acute stress in the HJS group, physiological stress did not dramatically differ according to job strain in this sample of relatively healthy nursing professionals who are able to do night-shift work. Further studies are warranted to establish the specific associations between job strain and sAA levels or salivary biomarker ratios. Knowledge of these associations would shed light on the specific mechanisms underlying the associations between long-term stress and negative health effects.

\section{Acknowledgment}

We thank Elise Koskenseppä for the participant recruitment, Nina Lapveteläinen and Riitta Velin for making the arrangements for the field measurements, Sirpa Hyttinen for the analysis of saliva samples, and Jaana Pentti and Hanna Kaisa Hyvärinen for statistical support. Language editing was done by Alice Lehtinen.

\section{Author Contributions}

KK contributed to design contributed to acquisition, analysis, and interpretation; drafted manuscript; critically revised manuscript; gave final approval; and agreed to be accountable for all aspects of work ensuring integrity and accuracy. MH contributed to conception and design, analysis, and interpretation; critically revised the manuscript; gave final approval; and agreed to be accountable for all aspects of work ensuring integrity and accuracy. MS contributed to conception and design and interpretation; critically revised manuscript; gave final approval; and agreed to be accountable for all aspects of work ensuring integrity and accuracy. HL contributed to design, analysis, and interpretation; critically revised manuscript; gave final approval; and agreed to be accountable for all aspects of work ensuring integrity and accuracy. AH contributed to design, acquisition, analysis, and interpretation; critically revised manuscript; gave final approval; and agreed to be accountable for all aspects of work ensuring integrity and accuracy. ME contributed to analysis and interpretation; critically revised manuscript; gave final approval; and agreed to be accountable for all aspects of work ensuring integrity and accuracy. MK contributed to conception and design and interpretation; critically revised manuscript; gave final approval; and agreed to be accountable for all aspects of work ensuring integrity and accuracy. JV contributed to conception, design, and interpretation; critically revised manuscript; gave final approval; and agreed to be accountable for all aspects of work ensuring integrity and accuracy. SP contributed to conception and design and acquisition, analysis, and interpretation; drafted the manuscript; critically revised the manuscript; gave final approval; and agreed to be accountable for all aspects of work ensuring integrity and accuracy.

\section{Declaration of Conflicting Interests}

The author(s) declared no potential conflicts of interest with respect to the research, authorship, and/or publication of this article.

\section{Funding}

The author(s) disclosed receipt of the following financial support for the research, authorship, and/or publication of this article: This study was supported by the Academy of Finland (projects 124473 and 132944), the Work Environment Fund (project 107156, grant 113334), the SalWe Research Program for Mind and Body (TEKES - The Finnish Funding Agency for Technology and Innovation, grant 1104/10), and the EU New OSH ERA Research
Programme. Mika Kivimäki is supported by a professorial fellowship from the Economic and Social Research Council, UK.

\section{References}

Ali, N., \& Pruessner, J. C. (2012). The salivary alpha amylase over cortisol ratio as a marker to assess dysregulations of the stress systems. Physiology \& Behavior, 106, 65-72. doi:10.1016/j.physbeh. 2011.10.003

Andrews, J., Ali, N., \& Pruessner, J. C. (2013). Reflections on the interaction of psychogenic stress systems in humans: The stress coherence/compensation model. Psychoneuroendocrinology, 38, 947-961. doi:10.1016/j.psyneuen.2013.02.010

Beck, A. T., Steer, R. A., \& Brown, G. K. (1996). Manual for the Beck Depression Inventory-II. San Antonio, TX: Psychological Corporation.

Chandola, T., Britton, A., Brunner, E., Hemingway, H., Malik, M., Kumari, M., ... Marmot, M. (2008). Work stress and coronary heart disease: What are the mechanisms? European Heart Journal, 29, 640-648. doi:10.1093/eurheartj/ehm584

Chandola, T., Heraclides, A., \& Kumari, M. (2010). Psychophysiological biomarkers of workplace stressors. Neuroscience \& Biobehavioral Reviews, 35, 51-57. doi:10.1016/j.neubiorev.2009.11.005

Chida, Y., \& Steptoe, A. (2009). Cortisol awakening response and psychosocial factors: A systematic review and meta-analysis. Biological Psychology, 80, 265-278. doi:10.1016/j.biopsycho.2008. 10.004

Clements, A. D., \& Parker, C. R. (1998). The relationship between salivary cortisol concentrations in frozen versus mailed samples. Psychoneuroendocrinology, 23, 613-616.

Clow, A., Thorn, L., Evans, P., \& Hucklebridge, F. (2004). The awakening cortisol response: Methodological issues and significance. Stress, 7, 29-37.

De Vente, W., Olff, M., Van Amsterdam, J. G., Kamphuis, J. H., \& Emmelkamp, P. M. (2003). Physiological differences between burnout patients and healthy controls: Blood pressure, heart rate, and cortisol responses. Occupational and Environmental Medicine, 60, i54-i61.

Dohrenwend, B. S., Krasnoff, L., Askenasy, A. R., \& Dohrenwend, B. P. (1978). Exemplification of a method for scaling life events: The Peri Life Events Scale. Journal of Health and Social Behavior, 19, 205-229.

Elovainio, M., Kivimäki, M., \& Vahtera, J. (2002). Organizational justice: Evidence of a new psychosocial predictor of health. American Journal of Public Health, 92, 105-108.

Federenko, I., Wust, S., Hellhammer, D. H., Dechoux, R., Kumsta, R., $\&$ Kirschbaum, C. (2004). Free cortisol awakening responses are influenced by awakening time. Psychoneuroendocrinology, 29, 174-184.

Fransson, E. I., Nyberg, S. T., Heikkilä, K., Alfredsson, L., Bacquer de, D., Batty, G. D., . . Kivimäki, M. (2012). Comparison of alternative versions of the job demand-control scales in 17 European cohort studies: The IPD-Work consortium. BMC Public Health, 12,62 .

Fries, E., Dettenborn, L., \& Kirschbaum, C. (2009). The cortisol awakening response (CAR): Facts and future directions. International 
Journal of Psychophysiology, 72, 67-73. doi:10.1016/j.ijpsycho. 2008.03.014

Fujimaru, C., Okamura, H., Kawasaki, M., Kakuma, T., Yoshii, C., \& Matsuishi, T. (2012). Self-perceived work-related stress and its relation to salivary IgA, cortisol and 3-methoxy-4-hydroxyphenyl glycol levels among neonatal intensive care nurses. Stress and Health, 28, 171-174. doi:10.1002/smi.1414

Fujiwara, K., Tsukishima, E., Kasai, S., Masuchi, A., Tsutsumi, A., Kawakami, N., ... Kishi, R. (2004). Urinary catecholamines and salivary cortisol on workdays and days off in relation to job strain among female health care providers. Scandinavian Journal of Work, Environment \& Health, 30, 129-138.

Groer, M., Murphy, R., Bunnell, W., Salomon, K., Van Eepoel, J., Rankin, B., ... Bykowski, C. (2010). Salivary measures of stress and immunity in police officers engaged in simulated critical incident scenarios. Journal of Occupational and Environmental Medicine, 52, 595-602. doi:10.1097/JOM.0b013e3181e129da

Gustafsson, K., Lindfors, P., Aronsson, G., \& Lundberg, U. (2008). Relationships between self-rating of recovery from work and morning salivary cortisol. Journal of Occupational Health, 50, 24-30.

Härmä, M., Kompier, M. A., \& Vahtera, J. (2006). Work-related stress and health risks, mechanisms and countermeasures. Scandinavian Journal of Work Environment \& Health, 32, 413-419.

Hintsanen, M., Elovainio, M., Puttonen, S., Kivimaki, M., Koskinen, T., Raitakari, O. T., \& Keltinkangas-Jarvinen, L. (2007). Effortreward imbalance, heart rate, and heart rate variability: The Cardiovascular Risk in Young Finns study. International Journal of Behavioral Medicine, 14, 202-212.

Houdmont, J., Kerr, R., \& Addley, K. (2012). Psychosocial factors and economic recession: The Stormont Study. Occupational Medicine, 62, 98-104. doi:10.1093/occmed/kqr216

Kajantie, E., \& Phillips, D. I. (2006). The effects of sex and hormonal status on the physiological response to acute psychosocial stress. Psychoneuroendocrinology, 31, 151-178.

Karasek, R. A., Brisson, C., Kawakami, N., Houtman, I., Bongers, P., \& Amick, B. (1998). The Job Content Questionnaire (JCQ): An instrument for internationally comparative assessments of psychological job characteristics. Journal of Occupational Health Psychology, 3, 322-355.

Karasek, R., \& Theorell, T. (1990). Healthy work: Stress, productivity and reconstruction of working life. New York, NY: Basic Books.

Karhula, K., Härmä, M., Sallinen, M., Hublin, C., Virkkala, J., Kivimäki, M., ... Puttonen, S. (2013). Association of job strain with working hours, shift-dependent perceived workload, sleepiness and recovery. Ergonomics, 56, 1640-1651. doi:10.1080/ 00140139.2013.837514

Kidd, T., Carvalho, L. A., \& Steptoe, A. (2014). The relationship between cortisol responses to laboratory stress and cortisol profiles in daily life. Biological Psychology, 99c, 34-40. doi:10.1016/j. biopsycho.2014.02.010

King, S. L., \& Hegadoren, K. M. (2002). Stress hormones: How do they measure up? Biological Research for Nursing, 4, 92-103.

Kirschbaum, C., Pirke, K. M., \& Hellhammer, D.H. (1993). The 'Trier Social Stress Test' - a tool for investigating psychobiological stress responses in a laboratory setting. Neuropsychobiology, 28, 76-81.
Kivimäki, M., Nyberg, S. T., Batty, G. D., Fransson, E. I., Heikkilä, K., Alfredsson, L., . . . Theorell, T. (2012). Job strain as a risk factor for coronary heart disease: A collaborative meta-analysis of individual participant data. Lancet, 380, 1491-1497. doi:10.1016/S01406736(12)60994-5

Kompier, M. A., Taris, T. W., \& van Veldhoven, M. (2012). Tossing and turning - insomnia in relation to occupational stress, rumination, fatigue, and well-being. Scandinavian Journal of Work Environment \& Health, 38, 238-246. doi:10.5271/sjweh.3263

Kudielka, B. M., Gierens, A., Hellhammer, D. H., Wust, S., \& Schlotz, W. (2012). Salivary cortisol in ambulatory assessment-some dos, some don'ts, and some open questions. Psychosomatic Medicine, 74, 418-431. doi:10.1097/PSY.0b013e31825434c7

Limm, H., Gundel, H., Heinmuller, M., Marten-Mittag, B., Nater, U. M., Siegrist, J., \& Angerer, P. (2011). Stress management interventions in the workplace improve stress reactivity: A randomised controlled trial. Occupational and Environmental Medicine, 68, 126-133. doi:10.1136/oem.2009.054148

Lindfors, P. M., Meretoja, O. A., Töyry, S. M., Luukkonen, R. A., Elovainio, M. J., \& Leino, T. J. (2007). Job satisfaction, work ability and life satisfaction among Finnish anaesthesiologists. Acta Anaesthesiologica Scandinavica, 51, 815-822. doi:10.1111/j.1399-6576. 2007.01343.x

Nater, U. M., La Marca, R., Florin, L., Moses, A., Langhans, W., Koller, M. M., \& Ehlert, U. (2006). Stress-induced changes in human salivary alpha-amylase activity-associations with adrenergic activity. Psychoneuroendocrinology, 31, 49-58.

Nater, U. M., Skoluda, N., \& Strahler, J. (2013). Stress biomarkers in behavioural medicine. Current Opinion in Psychiatry, 26, 440-445. doi:10.1097/YCO.0b013e328363b4ed

Pruessner, J. C., Kirschbaum, C., Meinlschmid, G., \& Hellhammer, D. H. (2003). Two formulas for computation of the area under the curve represent measures of total hormone concentration versus time-dependent change. Psychoneuroendocrinology 28, 916-931.

Pruessner, J. C., Wolf, O. T., Hellhammer, D. H., Buske-Kirschbaum, A., von Auer, K., Jobst, S., Kaspers, F., \& Kirschbaum, C. (1997). Free cortisol levels after awakening: A reliable biological marker for the assessment of adrenocortical activity. Life Sciences, 61, 2539-2549.

Rohleder, N., \& Nater, U. M. (2009). Determinants of salivary alphaamylase in humans and methodological considerations. Psychoneuroendocrinology, 34, 469-485. doi:10.1016/j.psyneuen.2008. 12.004

Rosenthal, T., \& Alter, A. (2012). Occupational stress and hypertension. Journal of the American Society of Hypertension, 6, 2-22. doi:10.1016/j.jash.2011.09.002

Rugulies, R. (2012). Studying the effect of the psychosocial work environment on risk of ill-health: Towards a more comprehensive assessment of working conditions. Scandinavian Journal of Work, Environment \& Health, 38, 187-191. doi:10.5271/sjweh.3296

Rydstedt, L. W., Cropley, M., Devereux, J. J., \& Michalianou, G. (2008). The relationship between long-term job strain and morning and evening saliva cortisol secretion among white-collar workers. Journal of Occupational Health Psychology, 13, 105-113.

Siegrist, J. (1996). Adverse health effects of high-effort/low-reward conditions. Journal of Occupational Health Psychology, 1, 27-41. 
Smith, C. S., Reilly, C., \& Midkiff, K. (1989). Evaluation of three circadian rhythm questionnaires with suggestions for an improved measure of morningness. Journal of Applied Psychology, 74, 728-738.

Steptoe, A. (2007). Psychophysiological contributions to behavioral medicine and psychosomatics. In J. T. Cacioppo, L. G. Tassinary, \& G. Berntson (Eds.). Handbook of psychophysiology (3rd ed., pp. 723-751). New York, NY: Cambridge University Press.

Steptoe, A., Cropley, M., Griffith, J., \& Kirschbaum, C. (2000). Job strain and anger expression predict early morning elevations in salivary cortisol. Psychosomatic Medicine, 62, 286-292.

Thayer, J. F., Yamamoto, S. S., \& Brosschot, J. F. (2010). The relationship of autonomic imbalance, heart rate variability and cardiovascular disease risk factors. International Journal of Cardiology, 141, 122-131. doi:10.1016/j.ijcard.2009.09.543

Thomas, C., Hertzman, C., \& Power, C. (2009). Night work, long working hours, psychosocial work stress and cortisol secretion in mid-life: Evidence from a British birth cohort. Occupational and Environmental Medicine, 66, 824-831. doi:10.1136/oem.2008. 044396
Vuori, M., Akila, R., Kalakoski, V., Pentti, J., Kivimäki, M., Vahtera, J., ... Puttonen, S. (2014) Association between exposure to work stressors and cognitive performance. Journal of Occupational and Environmental Medicine, 56, 354-360. doi:10.1097/JOM. 0000000000000129

Wingenfeld, K., Schulz, M., Damkroeger, A., Philippsen, C., Rose, M., \& Driessen, M. (2010). The diurnal course of salivary alpha-amylase in nurses: An investigation of potential confounders and associations with stress. Biological Psychology, 85, 179-181. doi:10.1016/j.biopsycho.2010.04.005

Wong, I. S., Ostry, A. S., Demers, P. A., \& Davies, H. W. (2012). Job strain and shift work influences on biomarkers and subclinical heart disease indicators: A pilot study. Journal of Occupational and Environmental Hygiene, 9, 467-477. doi:10.1080/15459624. 2012.693831

Woods, D. L., \& Mentes, J. C. (2011). Spit: Saliva in nursing research, uses and methodological considerations in older adults. Biological Research for Nursing, 13, 320-327. doi:10. $1177 / 1099800411404211$ 